\title{
Optical Azimuth Sensor for Indoor Mobile Robot Navigation
}

\author{
Keita Atsuumi and Manabu Sano \\ Graduate School of Information Sciences, Hiroshima City University \\ Japan
}

\section{Introduction}

A new type of azimuth angular sensor for an indoor mobile robot navigation is developed. This is a kind of optical sensors using infrared linear polarization. Since an angle is measured without contact, it is applicable to the navigation of an autonomous mobile robot. In the indoor environment, the navigation system by using GPS cannot be used. In dead reckoning, the accumulation of measurement error occurs a serious problem. If we use this sensor, we can measure a position only by installing one landmark all over the working space. We can acquire simultaneously not only the distance from a landmark but an angle of direction. Like a gyroscope or a geomagnetism sensor, the large drift depending on environment or time does not occur by this sensor. We make a prototype of the sensor based on this technique and conduct the measurement experiment. The accuracy of azimuth angle error is about $4 \%$ and the radial error is $93(\mathrm{~mm})$ at $3(\mathrm{~m})$ distance from the landmark.

An autonomous mobile robot needs to acquire self-position information at the time of moving in the working space. On actuators, such as a robot's mechanism and an arm with moving, there are many constraints in flexibility. Therefore, the position of the robot in the coordinate system fixed to working space and an azimuth angle are very important for moving in the working space. When working space is the outdoors, a self-position can be known with high precision using the positioning system (GPS) by an artificial satellite. It is also comparatively easy to measure geomagnetism using a flux-gate type or a semiconductor type magnetometric sensor, and to acquire an angle of direction. However, it is difficult to use such sensors in the indoor environment, for example, in an office, a factory and an inside of a tunnel and so on. Since an electric wave decreases the performance of the guidance control in the indoor autonomous mobile robot by existing the roof and the surface of a wall, the self-position measurement using GPS is difficult in those environment. The iron desk and the iron bookshelf which exist in the narrow space like office deteriorate the accuracy of a geomagnetism sensor. And though an angle of direction can be acquired using an angular velocity sensor (gyroscope), the error of measurement is accumulated with time. Although the method of marking beforehand on the floor and acquiring a self-position is technically easy, a lot of marks must be prepared manually, it is troublesome and inflexible. The specified of coordinates point in the working space is called a landmark. If working space with a two-dimensional spread is assumed, the point in coordinates cannot be 
determined a specified point only using the single landmark. The measuring method of the robot position using a infrared landmark was reported (N.Ushimi , M.Yamamoto et. al., 1999-2000). It is interesting that their method is simple in the structure and the position is derived from angles. Even though they use a landmark, it is disadvantage that they cannot determine the absolute azimuth. This research proposes a special idea to the landmark and shows how to acquire a position and an azimuth angle of direction simultaneously using a single landmark. The sensor system of the special infrared landmark is using linear polarization. The performance of the sensor is shown by the experiment.

\section{Basic Measureing Principle by Linear Polarizer}

\subsection{Linear Polarizer}

Generally, the random polarizing component is containing in the natural ray. Linear polarizer is a kind of optical filters that can pass through only the oscillating component of an e-vector along a single polarizing plane. There are two manufacturing methods: (a) Spattering method of the fine material fibers to a unique direction on the glass basis. In this case, it is superior to antienvironmental properties, the characteristics of wave lengths and the uniformity of the obtained polarizer ray. However it is difficult to handle and manufacture practically. (b) Stretching method of the colored high-polymer with the Iodine and unifying the orientation of the molecule and binding protecting by matrices. Even if this method has a disadvantage to minor changes of temperature and humidity, it is easy to cut and bind in the manufacturing process because it is thin, light and more flexible. In this study, we are focusing to the latter merit in particular, we realize a new angular sensor by using the layered polarizer and manufacturing to cone-like threedimensional shape.

\subsection{Polarizing Angle Measurement}

We use the modulation technique that the angular displacement can be measured as the angle of polarizing plane. We arrange the light source and the polarizer and the photodetector in Fig. 1. The polarizer(a) is fixed and the polarizer(b) is rotated around the axis. The light from the source is passed through both polarizers and arrived at the photo detector.

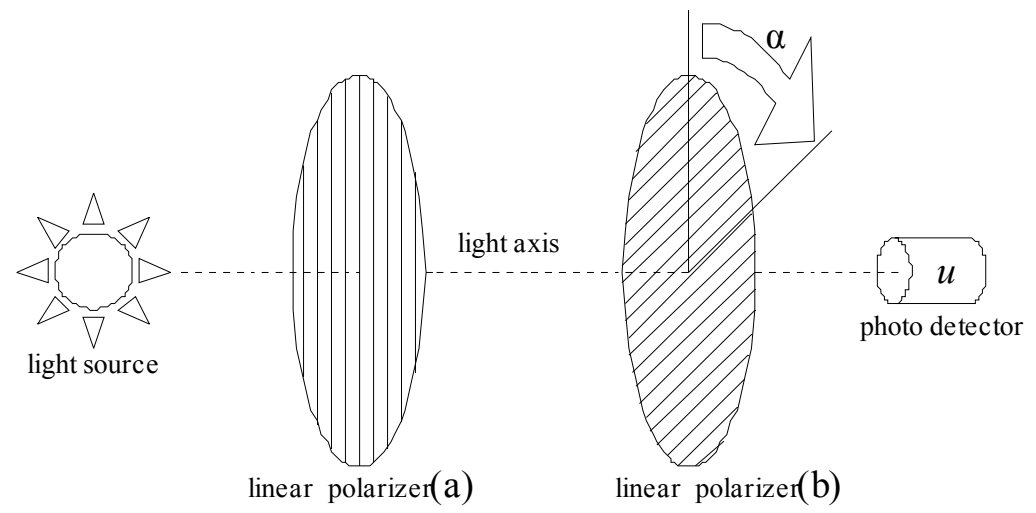

Fig. 1. Basic measuring principle by linear polarizer 
When we assume that the angle between the polarizer(a) and the polarizer(b) is a and the intensity of the light is $\mathrm{u}$, we can get the following expression,

$$
\mathrm{u}=\mathrm{A} \cos ^{2} \mathrm{a} \text {. }
$$

Here the $\mathrm{A}$ is an attenuation ratio which is positive constant less than 1.0 given by the material of polarizer or optical wavelength. The change of the polarizer(b) depends on the relative angle around the light axis between two polarizers. Since the polarizer(a) is located to the closer position with the measuring object and the polarizer $(b)$ is near to the sensor, the measuring resolution of the angle is not influenced principally by the arrangement with a distance of two polarizers. Many measuring techniques on the angle of polarized plane were reported and these techniques can be divided to two categories. One is the measuring method using some anglefixed polarizers and the photo detector coupled to the former polarizers. The other is the one using the rotated polarizer and the photo detector. We refered those works for measuring the angle of polarized plane; we apply the latter measuring technique to our system. We can measure the angle of polarizing plane on coming by the relative intensity of the arrived light to the photo detector. In our system, the polarizer(b) is rotated by using the small DC motor. If we calculate the phase from the timevaring angle change of the polarizer(b) and the time-varing change of the intensity of arrived light, we can estimate the angle on the polarizing plane. Thus, if we get the observing intensity of the light on the photo detector is $u^{*}$, the estimated value of the angle on the polarizing plane $\mathrm{a}^{*}$ is given by $(2 \mathrm{a}-\mathrm{c})$.

$$
\begin{gathered}
x^{*}=\int_{t=0}^{360} u^{*}(t) \cos (2 t) d t \\
y^{*}=\int_{t=0}^{360} u^{*}(t) \sin (2 t) d t \\
\alpha^{*}=\tan ^{-1} \frac{y^{*}}{x^{*}}
\end{gathered}
$$

\section{Cone Shape Linear Polarizer}

\subsection{Extension of Measurable Angle Range}

A certain kind of insect can return to a nest by assisting polarization in the sky. It is familiar that outdoors, weak polarization occurs in the sky by air correlate with sunrays. Such as an insect's behavior is applied to robot's action (D.Lambrinos et.al., 1997). At first, we have to understand the basic property of linear polarizing light. While the polarizer rotates one turn, same polarizing states appear twice. The range of an azimuth angle is $360(\mathrm{deg})$. On the contrary, the range of polarizing angle is $180(\mathrm{deg})$. This means that azimuth of $0(\mathrm{deg})$ and that of $180(\mathrm{deg})$ are undistinguished. Polarizing planes cannot discriminate the front surface and the rear surface. Since a linear polarizer is flat sheet in the original shape, there are some problems when we use it for the angle sensor. For solving this problem, we invent a new form of the linear polarizer for the angular sensor. We cut out the semi-circular sheet from the flat sheet of the linear polarizer. We make the cone-shaped linear polarizer from the semi-circular flat sheet by attaching each straight edge mutually around the center of the straight line as Fig. 2. We assume that the apex of the cone-wise plane is the point $\mathrm{O}$, the 
center axis is $\mathrm{Z}$, the angle along the cone-wise plane from the line $\mathrm{OA}$ to an arbitrary position $C$ is $\theta$, the rotating angle of the cone-wise plane around the Z-axis is $\varphi$, the angle between the line of vision (ray axis) and the ground is $\gamma$, and the inclined angle of the mother line is $\gamma_{0}$. We can obtain next formulas from the above geometrical relation,

$$
\begin{gathered}
\cos \varphi=\cos (2 \theta) \\
\sin \varphi=\cos \left(\gamma^{-} \gamma_{0}\right) \sin (2 \theta)
\end{gathered}
$$

If we assume the relation $\gamma=\gamma_{0}$, we can get

$$
\varphi=2 \theta
$$

In the above relation, the angle $\theta$ means the angle of the polarizing plane based on the line OA. Hence we can extend the polarizing angle $\theta$ in the range of $180(\mathrm{deg})$ to the rotating angle of the cone-wise plane $\varphi$ in the range of $360(\mathrm{deg})$. Since both angles are one-to-one correspondence, we can determine the azimuth angle uniquely. When the sharpness of the cone-wise plane is the angle $\gamma_{0}$, the extending shape of the cone-wise plane is determined uniquely as shown in Fig. 2. and we can get the next relation

$$
\gamma_{0}=30(\mathrm{deg}) \text {. }
$$

Then we can show that the rotating angle of the cone-wise plane around the Z-axis can be modulated as the angle of the polarizing plane around the ray axis. That is, if we set a light source inside the cone-wise polarizer and observe from outside the cone-wise plane, the angle of the observed polarizing plane is proportional to the rotating angle of the cone-wise plane.

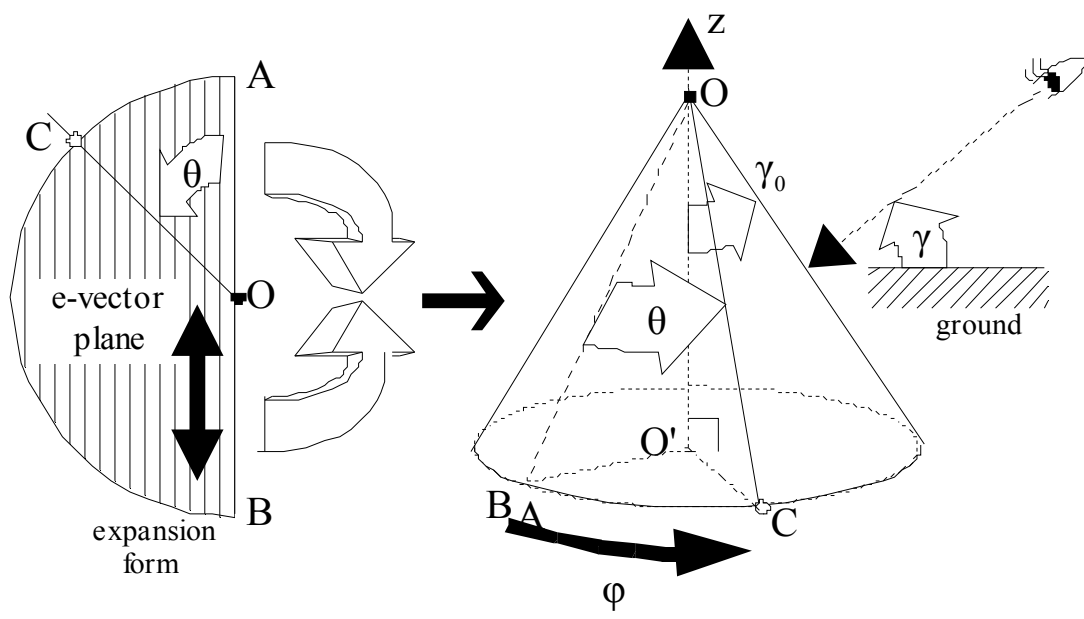

Fig. 2. 3-D conic structure made by flexible linear polarizer 


\subsection{Compensation of Discontinuity}

There is an adhered part that is parallel to the mother line in the manufactured cone-wise polarizer structurally. If we use a single cone-wise polarizer, sensing information is discontinuous and influenced polarizing properties at the junction. So we use two cone-wise polarizers as shown in Fig. 3. and assign them at distorted angles each other for compensating discontinuity.
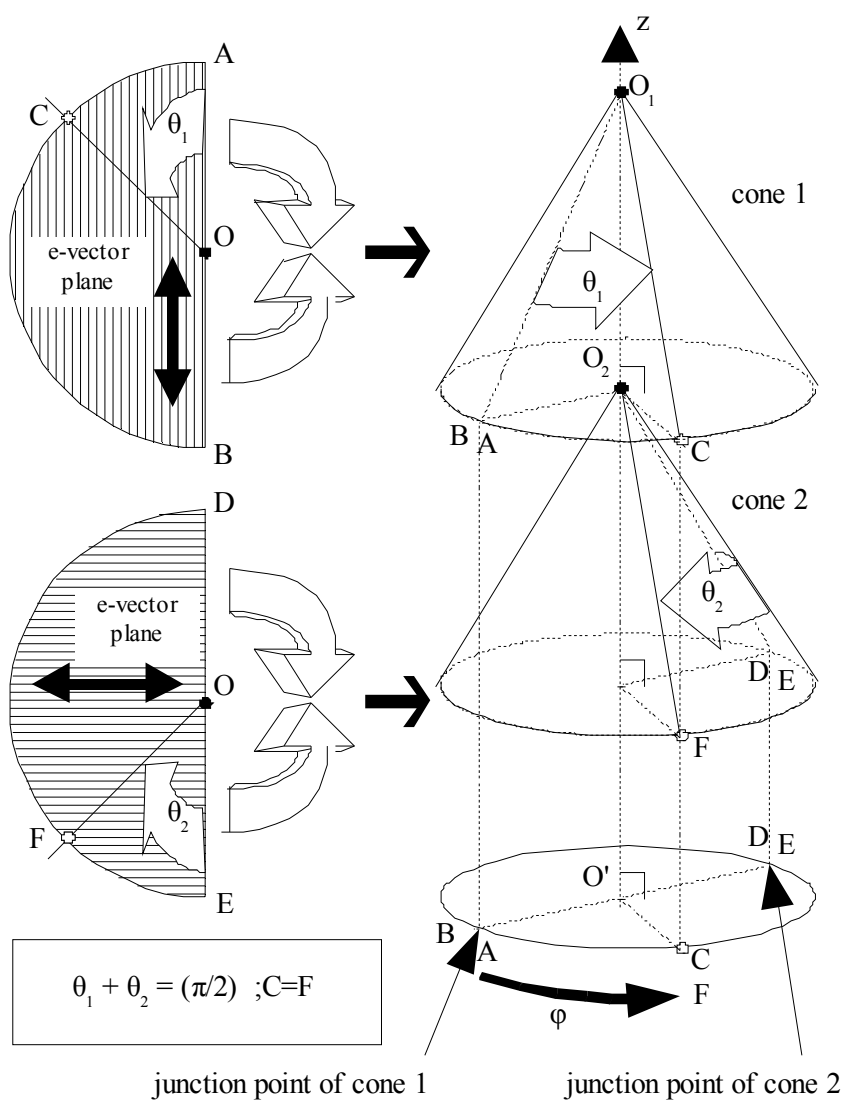

Fig. 3. Elimination of constructional discontinuity

\section{Measuring Principle of Distance}

\subsection{Distance Determination by Elevation Angle}

At indoor environment, we image the working space with the constant distance from the floor to the ceiling. We set an infrared landmark to a ceiling and we assume that the landmark is located at the arbitrary ceiling in the working space. The light coming from a landmark is correctly caught on the directive principal axis by the sensor attached to the robot. If it assumes that the height $\mathrm{H}$ from floor to the ceiling is constant, the horizontal 
distance from the transmitter to the receiver can be calculated from the elevation of the receiver as shown in Fig. 4. If the elevation angle $\beta$ is measured, the horizontal distance $\mathrm{L}$ between a transmitter and a receiver will be obtained by

$$
L=\frac{H}{\tan \beta} .
$$

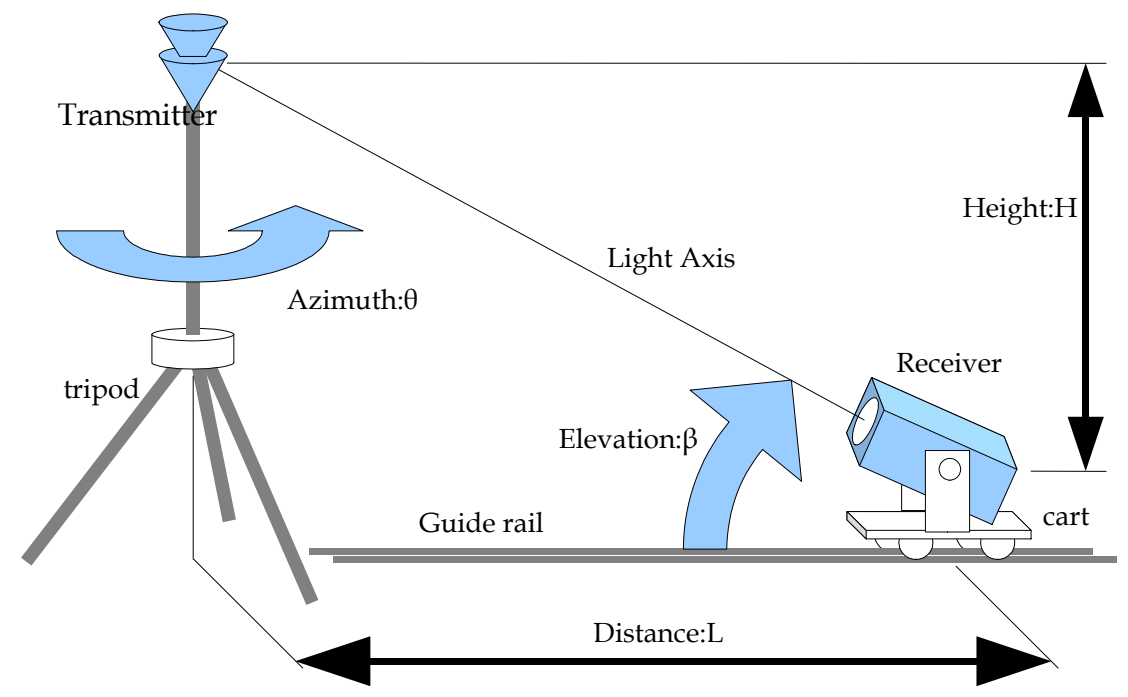

Fig. 4. Experimental setup

\subsection{Elevation Angle Measurement using Accelerometer}

An elevation angle is measured from the plane of the floor. With the ideal level, a floor is not always horzontal but there is an inclination and unsmoothness. When the cart of the mobile robot with the sensor may incline, the measured result of the elevation angle might be included some errors. So we measure the elevation angle on the basis of gravity acceleration. We measure gravity acceleration using a semiconductor-type acceleration sensor and acquire an elevation angle from the ratio of gravity acceleration which acts on each axis. If the robot is stationary, downward gravity acceleration will act on a sensor. An acceleration sensor has specific axes which shows higher sensitivity. In this research, we call them sensitivity axes. 


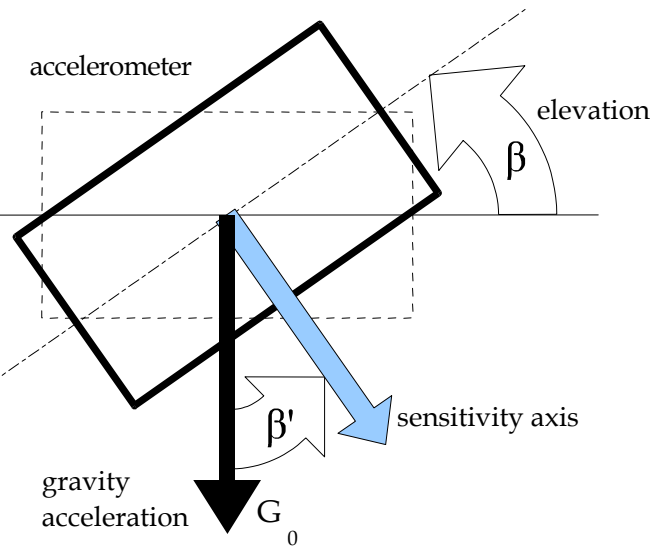

Fig. 5. Accelerometer and sensitivity axis

We set a sensitivity axis perpendicular to downward direction with $\beta^{\prime}$ as the preparation of measurements as shown in Fig. 5. An output voltage from gravity acceleration $V_{\text {out }}$ which acts along a single sensitivity axis is expressed in the following

$$
\mathrm{V}_{\text {out }}=\mathrm{K}_{\mathrm{s}} \mathrm{G}_{0} \cos \beta^{\prime}+\mathrm{V}_{\text {offset }} \text {. }
$$

Here $K_{s}$ is the sensor gain, $G_{0}$ is constant gravity accerelation and $V_{\text {offset }}$ is offset voltage of the sensor which adjust to zero in advance. Differentiating (7) about $\beta^{\prime}$, we get

$$
V_{\text {diff }}=-K_{s} G_{0} \sin \beta^{\prime}
$$

We know, the closer a sensitivity axis approaches vertically from horizontal axis, the worse the sensitivity of an acceleration sensor becomes.

\subsection{Improvement of the measureing precision}

When the elevation angle $\beta$ in eq.(6) is include the measuring error $\Delta \beta$, we get

$$
L+\Delta L=\frac{H}{\tan (\beta+\Delta \beta)}=H\left(\frac{1-\tan \beta \tan \Delta \beta}{\tan \beta+\tan \Delta \beta}\right) .
$$

By Eqs.(6) and (9), the distance error $\Delta \mathrm{L}$ is shown as follows

$$
\Delta L=H\left(\frac{1-\tan \beta \tan \Delta \beta}{\tan \beta+\tan \Delta \beta}-\frac{1}{\tan \beta}\right)
$$


In the height $\mathrm{H}$ is constant value $(\mathrm{H}=2.0(\mathrm{~m}))$, the relation between distance error and elevation angle error is shown as Fig. 6, in the case of $d=0.1,1,5$ and 10 (m).

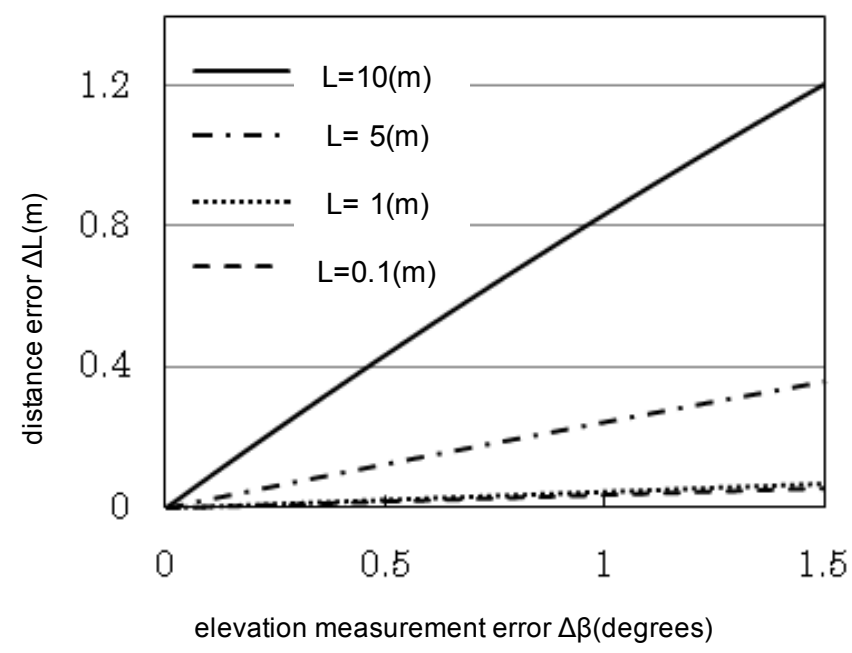

Fig. 6. Distance error vs elevation measurement error

Gravity acceralation on the stationally body is always constantly downward $1.0(\mathrm{G})$. If we assume components of the acceleration $\mathrm{X}_{\mathrm{G}}(\mathrm{G})$ and $\mathrm{Y}_{\mathrm{G}}(\mathrm{G})$, with the inclination angle $\beta(\mathrm{deg})$ of mutually orthogonal principle axes of accelerations, $\boldsymbol{\beta}^{*}$ is satisfied with the following

$$
\begin{aligned}
& \beta_{x}{ }^{*}=\cos ^{-1}\left(\frac{V_{\text {out } x}}{K_{\text {sx }} G_{0}}\right) . \\
& \beta_{y}{ }^{*}=\cos ^{-1}\left(\frac{V_{\text {out } y}}{K_{\text {sx }} G_{0}}\right) .
\end{aligned}
$$

When we measure the acceleration among which axis, we get the elevation on that axis. In the elevation angle measurement of using single axis, the measureing presition is remarkably decreased by the non-linearity of the trigonometric function at the specified angles.

The sensitivity of the gravity acceleration affects on that of the elevation angle at the proximity of the angle which the principle axis is parallel to the vartical axis. We compensate the elevation angle measurement by using multi axes in the following two approaches so that we consider the angle range of confirming more presize measurement.

(a) Right angle switching method; for excluding the angle range of principle axes with the remarkably worth precision, we use the single axes of more suitable axis. Such the angle $\beta^{*}(\mathrm{deg})$ is including the range of $0 \leq \beta<45,135 \leq \beta<225$ and $315 \leq \beta<360$, we use the angle on the $\mathrm{X}$-axis and otherwise we use the angle on the $\mathrm{Y}$-axis. i.e. 


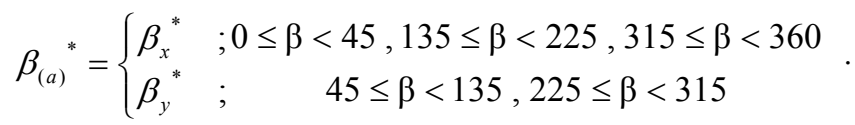

(b) Weighting method; the way of measureing angle without switching axis that we put the more weight as more principle axis is closer to horizontal direction and vice versa. If we make the voltage $V_{x}(V), V_{y}(V)$ and the angle of $\beta_{\mathrm{x}}{ }^{*}(\mathrm{deg}), \beta_{\mathrm{y}}{ }^{*}(\mathrm{deg})$ of each axis, we can get the weighting average as follows,

$$
\beta_{(b)}^{*}=\beta_{x}^{*}\left(\frac{V_{y}}{V_{x}+V_{y}}\right)+\beta_{y}^{*}\left(\frac{V_{x}}{V_{x}+V_{y}}\right) .
$$

We use the electric capacity type 3-axes semiconductor acceleration sensor (Kionix KXM521050). Sensitivity axes of this sensor cross orthogonal mutually. We measured the elevation angle using two of three sensitivity axes $V_{\text {out } x}$ and $V_{\text {out } y}$.

An $\mathrm{X}$-axis positive direction is defined as 0 (deg), Y-axis positive direction is 90 (deg) and Zaxis is perpendicular to the $X-Y$ plane. That is used as rotation axis in this experiment. Next, we adjust offset and gain of an accelerometer that $X$-axis output voltage $V_{\text {out } x}$ to $0(V)$ when the $\mathrm{X}_{0}$ axis is $0(\mathrm{deg}), \mathrm{Y}$-axis output voltage $\mathrm{V}_{\text {out y }}$ to $0(\mathrm{~V})$ when the $\mathrm{X}$-axis is $90(\mathrm{deg})$. We regard the angle set by the angle-setup apparatus as the true value in $\mathrm{X}$-axis direction. Then we adjust each 5(deg) in the range of $0 \sim 355(\mathrm{deg})$ and we compare the error between the angle calculated from accelerometer output (angle measurement) and that of the evaluated value. In addition, we compare and evaluate on two ways that uses two axes.

\begin{tabular}{|c|c|c|c|c|}
\hline method & $\beta^{*}$ only & $\beta^{*}$ only & Right angle switching & Weighting \\
\hline maximum error $(\mathrm{deg})$ & 1.479 & 2.860 & 0.321 & 0.294 \\
\hline variance & $6.37 \mathrm{e}-2$ & $1.88 \mathrm{e}-1$ & $3.26 \mathrm{e}-2$ & $5.49 \mathrm{e}-2$ \\
\hline S.D. & $2.52 \mathrm{e}-1$ & $4.33 \mathrm{e}-1$ & $1.81 \mathrm{e}-1$ & $2.34 \mathrm{e}-1$ \\
\hline
\end{tabular}

Table 1. Relationship of measurement error

The table1 summarize the angle error of the masured angle and the true one by calculating measured data on one axis or two axes. In all items, the two-axis measureing accuracy is better than that of the data by single axis. Additionally in two-axis measurement by using a weighting method, the maximum error seems to be improved. By using the weighting method, the maximum error in the best case is suppressed under the $1 / 10$ value when compared with the single axis. The maximum elevation error obtained by weighting method is $0.294(\mathrm{deg})$. If we calculate the distance error from this result, eventhough the distance $\mathrm{L}$ from the experimental apparatus in Fig. 4 to the target point is $10(\mathrm{~m})$, the error of the distance is about $0.3(\mathrm{~m})$. Therefore, this measuring apparatus and technique can confirm the high precision on the distance measurement. We employed the weighting method for distance measurement. 


\section{Experimental Setup}

\subsection{Introduction}

The proposed sensor consists of two parts. One is the transmitter which emits polarizedmodulating infrared rays. Another is the receiver which demodulates infrared rays from the transmitter. Thus we can acquire the heading of the receiver's azimuth. By using the transmitter as the landmark, we measure a self-position and absolute azimuth of the receiver. A schematic view of an experimental setup is shown in Fig. 4. The transmitter is attached to a tripod at the height of $1700(\mathrm{~mm})$ from the floor. The vertex direction of conic polarizer corresponds to downward. Since the transmitter can rotate arround a perpendicular axis, it can set arbitrary azimuth angle. The receiver is installed to the cart on the straight rail for setting arbitarary horizontal distance. Setting the height of the receiver to $200(\mathrm{~mm})$ with the cart, we can get the height of $\mathrm{H}=1500(\mathrm{~mm})$ between the transmitter and the receiver.

\subsection{Transmitter}

The transmitter plays the most important role in this sensor. It consists of an infrared LED array, a pulse generating circuit, and a conic linear polarizer. If the LED is driven by a narrow duty pulse with a subcarrier frequency of $1(\mathrm{MHz})$, momentary optical power can be increased and we make the signal easy to distinguish from the disturbance light which comes out of lighting apparatus. The infrared rays emitted from LED have strong directivity, and the light intensity which comes out of single LED is weak. In order to keep the strong light intensity, seven LEDs have been arranged over 1 turn. Since the polarizing plane is discontinuous at the jointed line on the cone, we want to shut off the light at this point. We employed a special idea and made a sophisticated device that we used to combine two modules with mutually different direction of jointed line. The block diagram of the transmitter is shown in Fig. 7. Actual size of conic linear polarizer is about $50(\mathrm{~mm})$ diameter.
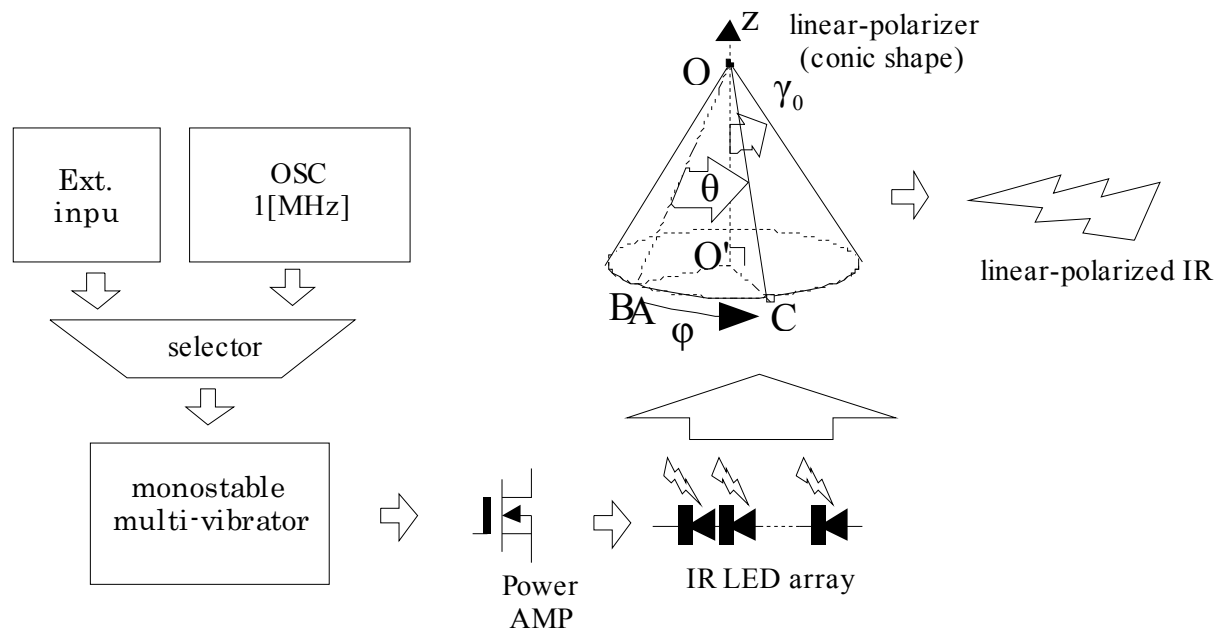

Fig. 7. Block diagram of the transmitter 


\subsection{Receiver}

A receiver is constituted by a convex lens, a rotating light polarizer, a photo detector, a preamplifier, and an AM receiving circuit. The mechanical structure of a receiver is shown in Fig. 8. The light coming from the transmitter is first condensed with a convex lens about $35(\mathrm{~mm})$ diameter. Next, the light is passed through the polarizer attached to the small DC motor, which made an amplitude modulation (AM) signal, and a photo detector. The motor has a rotation-synchronized pulse generator. The light which entered into the photo detector is changed into an electric signal, and is inputted into the AM receiving circuit through a preamplifier. The AM receiving circuit is equivalent to the AM middle wave radio in of a superheterodyne system. Thus, the light signal is convert to the phase between the synchronized pulse and the sine wave depend on the angle of polarizing plane. The signal frequency is twice of a motor speed as an AF band approximately $400(\mathrm{~Hz})$. A received signal is taken into PC from A/D conversion through after a low pass filter. Based on the pulse outputted from a motor, the phase of a received sine wave-like signal is proportional to the angle of the linear polarization of the light from the transmitter. Therefore, we will be obtained that the angle around the perpendicular axis of a transmitter by calculate the phase difference.

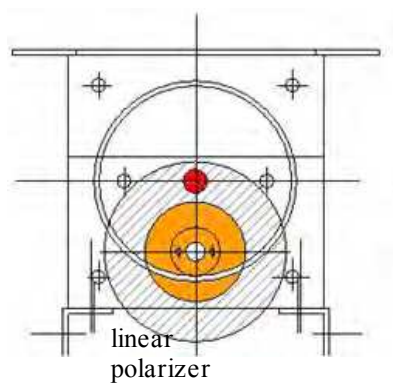

front view

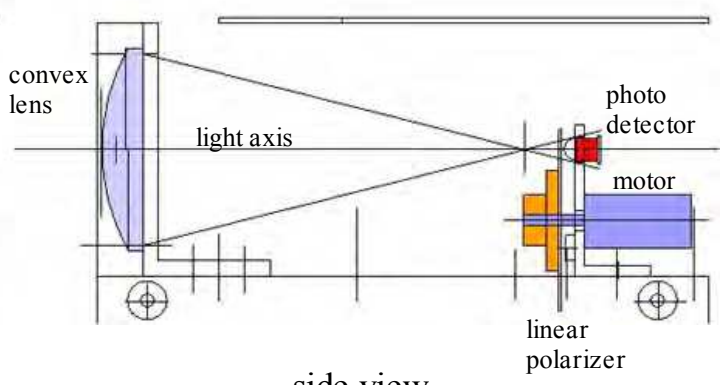

side view

Fig. 8. Mechanical structure of the receiver (fragment)

\section{Experimental Result and Discussion}

\subsection{Azimuth Measurement}

A transmitter is rotated every 10 degrees and azimuth angles at specified ones among 1 turn are measured. The distance from a transmitter to a receiver is influenced by the measuring error of angles. When we change the distance L as the Fig. 4 from $1000(\mathrm{~mm})$ to $3000(\mathrm{~mm})$ at each $500(\mathrm{~mm})$, the measured results of the angle is shown in Fig. 9. Also Fig. 10 shows the measurement angle error. The alignment relation is obtained within $4 \%$ at all over the distance range. When the linear polarized light is transmitted inside of the free space where a refractivity does not change, a polarizing plane is maintained. Therefore, angle measurement is not influenced even if distance changes theoretically. However, if the distance from a transmitter to a receiver increases, as a result of a signal declination, in a long distance, the $\mathrm{S} / \mathrm{N}$ ratio may deteriorate and angle measurement may be affected. The receiver for an experiment rotates the polarizer using the motor, and can obtain the angle of 
polarization from the phase. If the rotation speed of a motor changes, since the generated delay in LPF will change relatively, the measurement accuracy of a phase deteriorates.

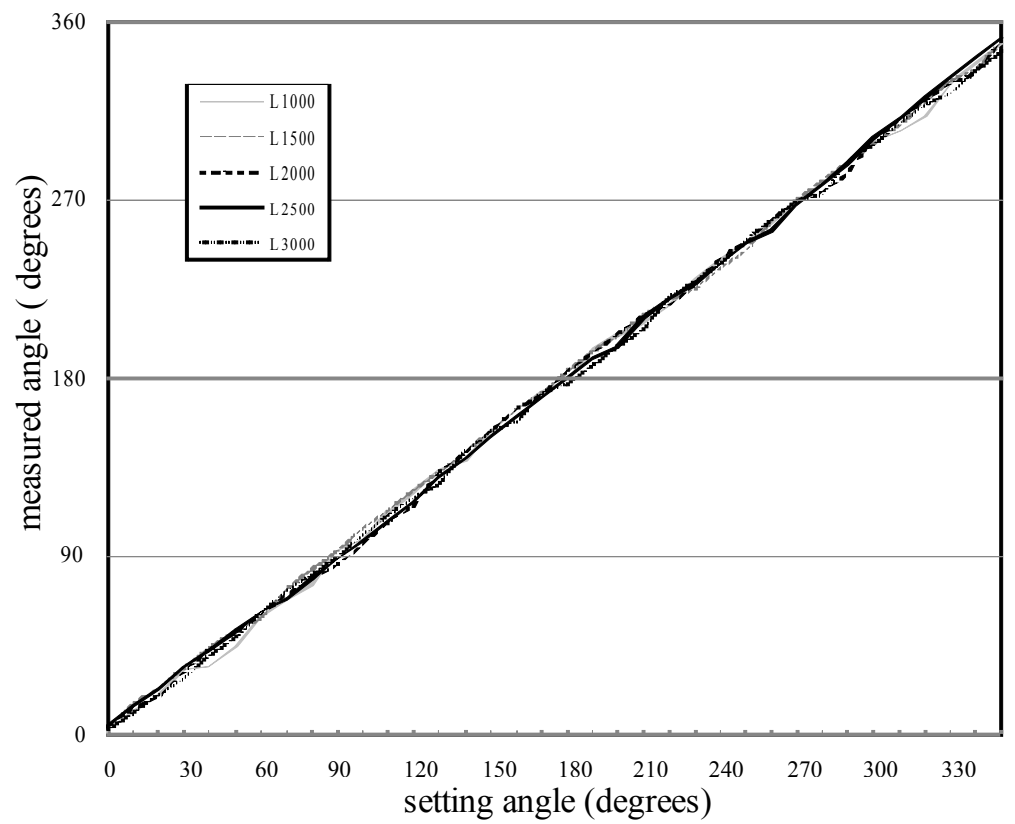

Fig. 9. Measured angle vs set azimuth angle (L:mm)

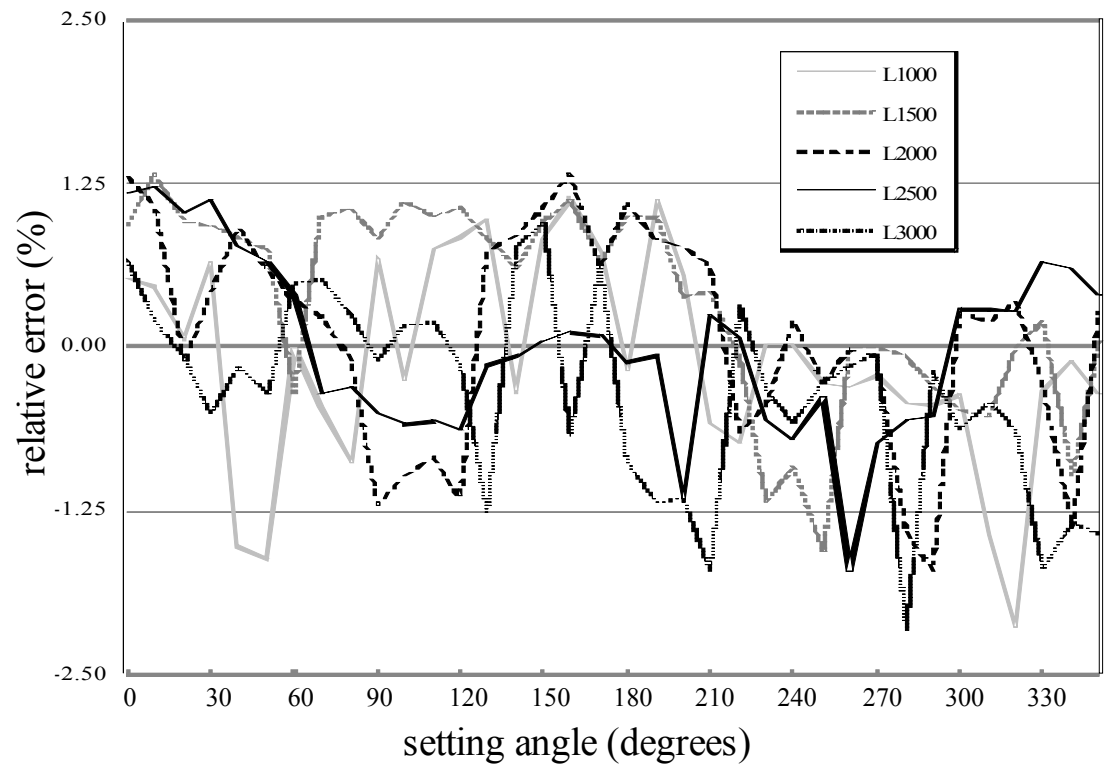

Fig. 10. Measurement error of azimuth angle (L:mm) 
A polarizing plate is not moved mechanically but the detecting method of an angle from the strength of the signal from two or more sensors is also discussed (see references). While, in order to receive the light of a transmitter from several meters away, we have to set light axis precisely. It is difficult to configure two or more sensors with same properties exactly. If we employ the two or more divided type monolithic photodiode, it may solve to the problem to some extent. However, we have to attach the polarizing plate adjusted to the angle in front of each element. Our system should be considered as only one optical sensor in total. If the speed of a motor can be stabilized more accurately we expect the measurement accuracy of the direction angle to increase.

\subsection{Localization Measurement}

Fig. 11 depicts the distance measurement result. Relation between setting distance and measured one is linear. The latter shows less than the former. In this experiment, the absolute maximum error is $93(\mathrm{~mm})$ at set distanse of $3000(\mathrm{~mm})$. Finally, we get Fig. 12 which is whole result of the experiment. This $r-\theta$ plot illustrates that estimated position of a mobile robot using our sensor system. Of course, the center is a landmark.

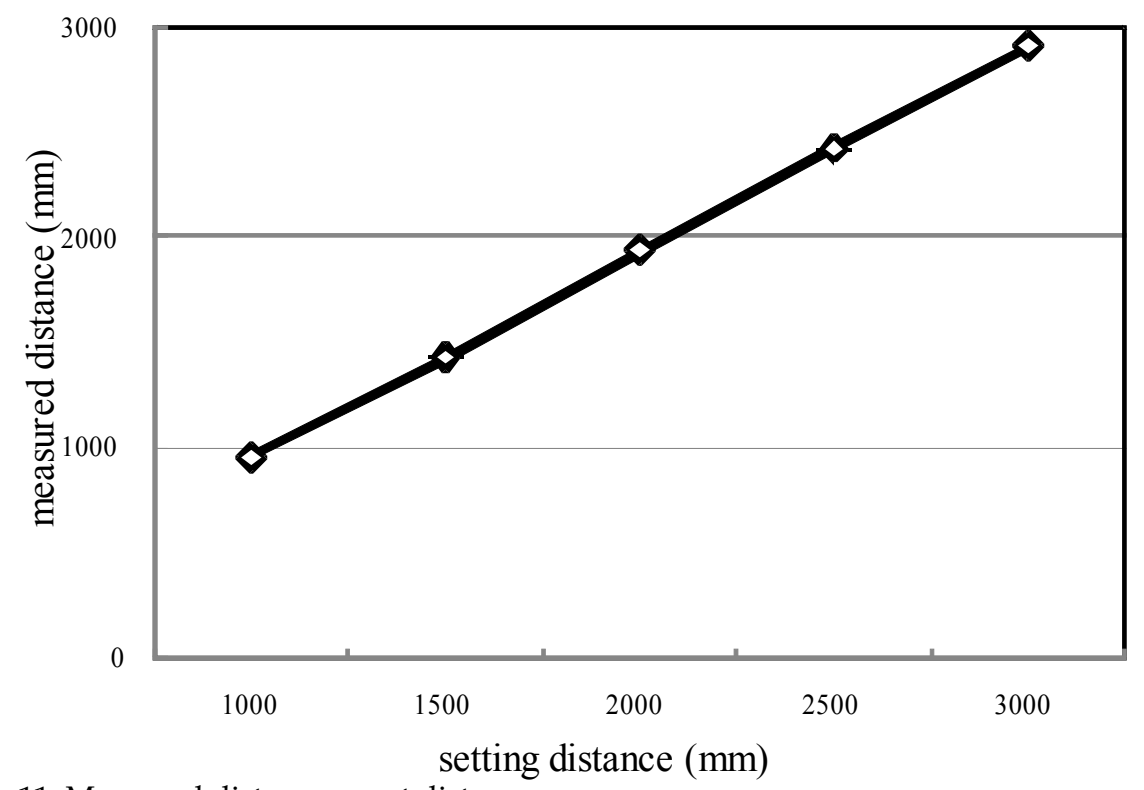

Fig. 11. Measured distance vs set distance 


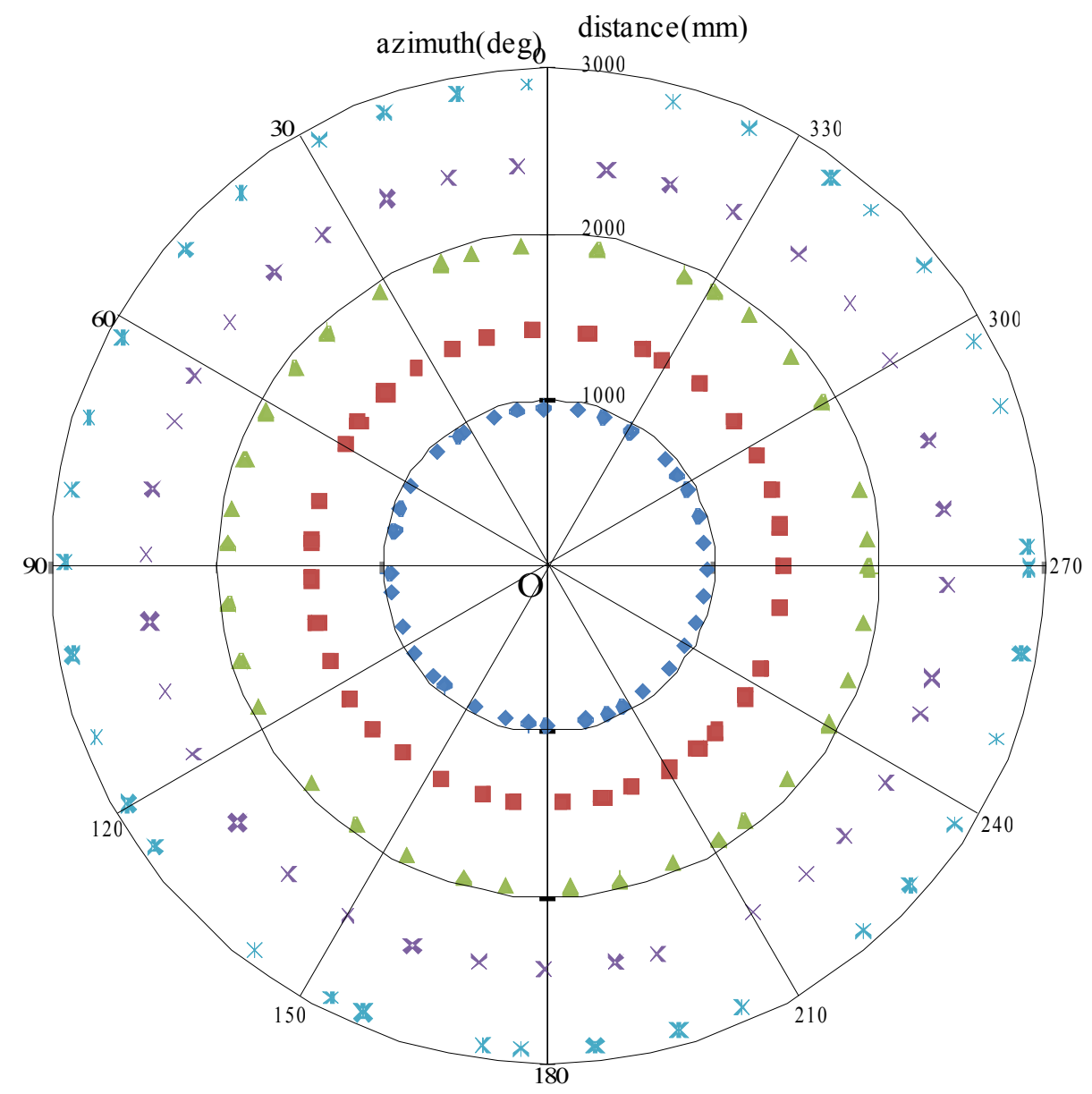

Fig. 12. Localization result of our sensor system.

\section{Conclusion}

We can acquire simultaneously both the azimuth angle and the distance from the target position by using a single landmark. This sensing system is constructed by combination of the optical sensor using infrared linear polarizer which developed by authors and the commercial semiconductor type of acceleration sensor. By using a semiconductor acceleration sensor, experiments on the elevation angle were measured based on the direction of gravity. It is very useful to acquire the information on the position and the angle in the indoor environment. 


\section{References}

D.Lambrinos, M.Maris, H.Kobayashi, T.Labhart, R.Pfeifer and R.Wehner (1997), "An Autonomous Agent Navigating with a Polarized Light Compass", Adaptive behavior, Vol.6-No.1,pp.131-161

K.Atsuumi, M.Hashimoto and M.Sano(2008). "Optical Azimuth Sensor for Indoor Mobile Robot Navigation", The 2008 International Conference on Computer Engineering \& Systems(ICCES'08), ISBN:978-1-4244-2116-9, Cairo, Egypt.

M.Yamamoto, N.Ushimi and A.Mohri(1999-Mar), "Navigation Algorithm for Mobile Robots using Information of Target Direction", Trans.JSME,Vol.65-No.631,pp.1013-1020. (in Japanese)

N.Ushimi, M.Yamamoto and A.Mohri(2000-Mar), "Development of a Two Degree-of-Freedom Target Direction Sensor System for Localization of Mobile Robots", Trans.JSME, Vol.66No.643, pp.877-884. (in Japanese)

Japanese patent No.2001-221660(2001) (in Japanese)

Japanese patent No.H08-340475(1996) (in Japanese) 


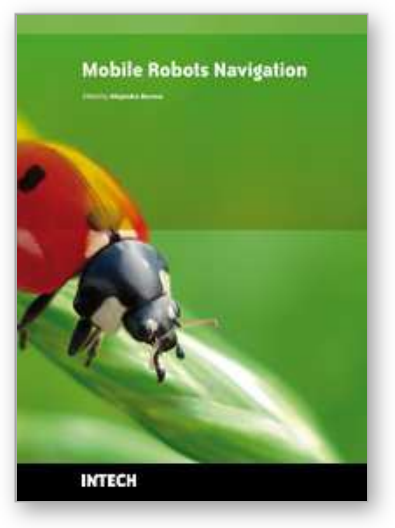

\author{
Mobile Robots Navigation \\ Edited by Alejandra Barrera
}

ISBN 978-953-307-076-6

Hard cover, 666 pages

Publisher InTech

Published online 01, March, 2010

Published in print edition March, 2010

Mobile robots navigation includes different interrelated activities: (i) perception, as obtaining and interpreting sensory information; (ii) exploration, as the strategy that guides the robot to select the next direction to go; (iii) mapping, involving the construction of a spatial representation by using the sensory information perceived; (iv) localization, as the strategy to estimate the robot position within the spatial map; (v) path planning, as the strategy to find a path towards a goal location being optimal or not; and (vi) path execution, where motor actions are determined and adapted to environmental changes. The book addresses those activities by integrating results from the research work of several authors all over the world. Research cases are documented in 32 chapters organized within 7 categories next described.

\title{
How to reference
}

In order to correctly reference this scholarly work, feel free to copy and paste the following:

Keita Atsuumi and Manabu Sano (2010). Optical Azimuth Sensor for Indoor Mobile Robot Navigation, Mobile Robots Navigation, Alejandra Barrera (Ed.), ISBN: 978-953-307-076-6, InTech, Available from: http://www.intechopen.com/books/mobile-robots-navigation/optical-azimuth-sensor-for-indoor-mobile-robotnavigation

\section{INTECH}

open science | open minds

\section{InTech Europe}

University Campus STeP Ri

Slavka Krautzeka 83/A

51000 Rijeka, Croatia

Phone: +385 (51) 770447

Fax: +385 (51) 686166

www.intechopen.com

\section{InTech China}

Unit 405, Office Block, Hotel Equatorial Shanghai

No.65, Yan An Road (West), Shanghai, 200040, China

中国上海市延安西路65号上海国际贵都大饭店办公楼405单元

Phone: +86-21-62489820

Fax: $+86-21-62489821$ 
(C) 2010 The Author(s). Licensee IntechOpen. This chapter is distributed under the terms of the Creative Commons Attribution-NonCommercialShareAlike-3.0 License, which permits use, distribution and reproduction for non-commercial purposes, provided the original is properly cited and derivative works building on this content are distributed under the same license. 